Images in clinical medicine

\title{
Mb. Bowen and squamous-cell carcinoma on the eczematous skin
}

\author{
Boris Jančar \\ Department of Radiation Oncology, Institute of Oncology, Ljubljana, Slovenia
}

A 64-year-old female patient had eczema on the forefinger of the left hand for 12 years. As the appearance of the lesion changed, biopsy was performed. Histological examination confirmed $\mathrm{Mb}$. Bowen that further developed into squamous-cell carcinoma on the eczematous skin that covered almost the entire circumference of the finger (Figures 1a, 1b). Surgery was considered too mutilating, whereas irradiation was technically too difficult to perform and late consequences on the vessels of the finger were considered to be too severe.

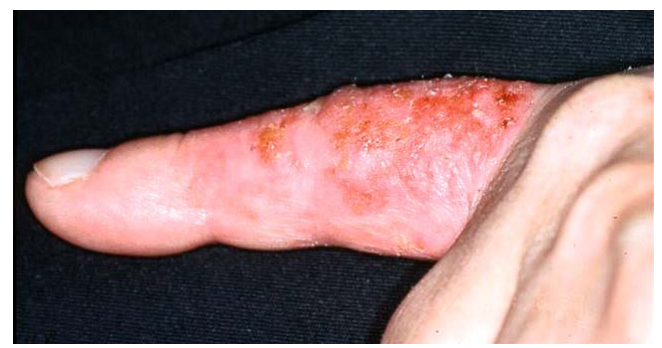

Figure 1a. Squamous-cell carcinoma on the eczematous skin.

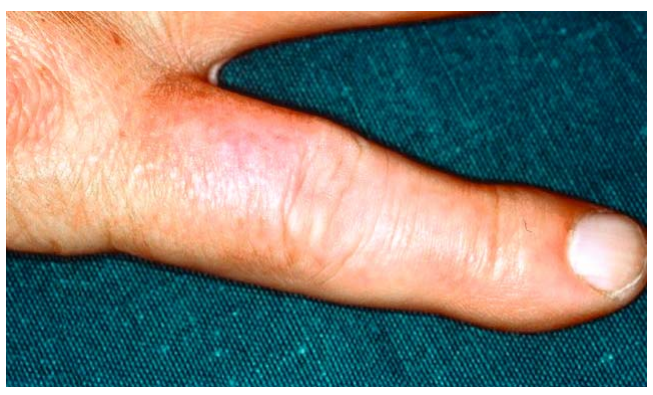

Figure 2. A year after therapy, only erythema of the skin was still present.
The patient was treated with interferon (altogether 6 intralesional applications; 3 times a week) and vitamin A (30 mg/day; 3 weeks).

A year after the completed therapy, only erythema of the skin was still present, while the skin was smooth and soft (Figure 2). Five years after the completed therapy, the treated skin on the forefinger of the left hand did not differ from the untreated healthy skin (Figure 3).

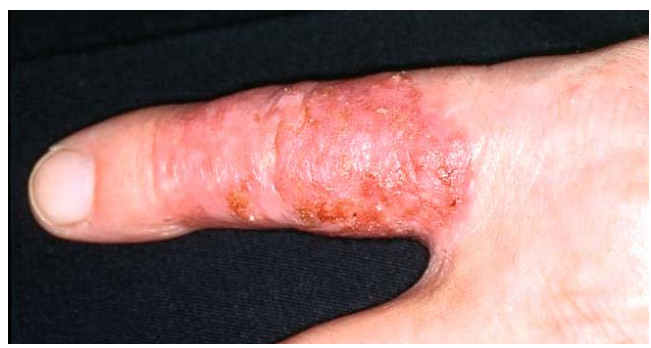

Figure 1b. Squamous-cell carcinoma on the eczematous skin.

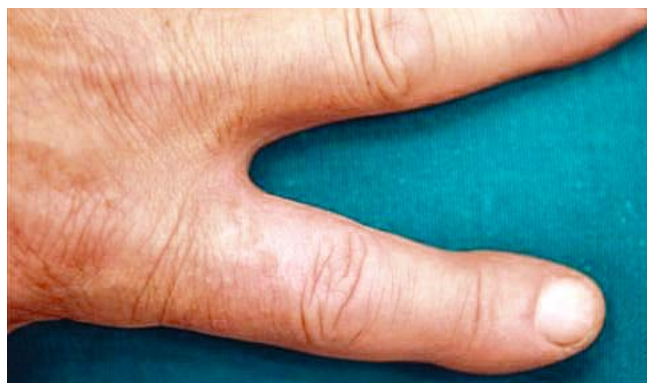

Figure 3. Five years after therapy, a complete remission of carcinoma was seen. 